Meta

Journal des traducteurs

Translators' Journal

\title{
Les présuppositions du discours : un dilemme pour le traducteur
}

\section{Enriqueta Ribé et Miguel Olivera}

Volume 24, numéro 1, mars 1979

La traduction juridique

URI : https://id.erudit.org/iderudit/003948ar

DOI : https://doi.org/10.7202/003948ar

Aller au sommaire du numéro

Éditeur(s)

Les Presses de l'Université de Montréal

ISSN

0026-0452 (imprimé)

1492-1421 (numérique)

Découvrir la revue

Citer cet article

Ribé, E. \& Olivera, M. (1979). Les présuppositions du discours : un dilemme pour le traducteur. Meta, 24(1), 130-140. https://doi.org/10.7202/003948ar d'utilisation que vous pouvez consulter en ligne. 


\section{Les présuppositions du discours: un dilemme pour le traducteur}

\section{La relativité sémantique du lexique juridique}

Dans le domaine juridique constitutionnel, politique ou administratif, la traduction pose naturellement le problème de l'équivalence entre institutions organisées sur des principes, normes et usages propres à chaque société. Des mots courants et sémantiquement bien délimités pour les citoyens d'un régime parlementaire, tels que «ministre», par exemple, comportent des connotations diverses pour ceux d'une république présidentielle, et les écarts seront multipliés pour les sujets d'une monarchie absolue. Les dénominations des responsabilités publiques, la portée des attributions des fonctionnaires, les droits et les devoirs des individus dans leurs rapports avec les organes de l'État, les juridictions de l'autorité et la distribution du pouvoir, ce sont là des notions qui doivent être souvent expliquées dans une traduction destinée à des lecteurs d'une autre société, si l'on veut que ceux-ci en retirent une connaissance minimale du fonctionnement des institutions. Et si cela arrive fréquemment entre nations voisines, héritières des traditions communes et dans le cadre d'une même civilisation (comme peuvent l'être, par exemple, les membres de la communauté européenne), le problème devient plus complexe à mesure que l'horizon culturel s'élargit. On intègre alors dans le cadre de référence - comme l'exige la dimension planétaire du monde contemporain - les civilisations «périphériques » d'Asie, d'Afrique et d'Amérique latine, des régions où les modèles montrent une mixture complexe d'institutions autochtones submergées sous l'implantation colonialiste européenne, le tout bouleversé par les mouvements nationaux de libération et les processus de modernisation.

La nécessité de préciser et d'expliciter le sens que les termes du vocabulaire juridique-institutionnel peuvent acquérir dans un contexte particulier se double du défi lancé par la distorsion sémantique née des changements historiques et de l'utilisation idéologique du langage courant. D'une part, le maire qui dirigeait le corps municipal dans les «villes de commune » françaises sous l'Ancien Régime n'a pas, naturellement, les mêmes traits sémantiques, du point de vue de son pouvoir politique, que le primum inter pares choisi par le conseil municipal après la Révolution de 1789 , et les connotations politiques du mot dans son sens contemporain d' "agent du pouvoir central dans chaque arron- 
dissement de Paris » ont évidemment changé l'année dernière à partir de l'élection du maire de Paris au suffrage universel. D'autre part, le mot démocratie, par exemple, n'a non plus le même sens, comme l'ont déjà signalé autant des linguistes que des écrivains de fiction, quand il est utilisé par différents groupes sociaux dans des situations historiques différentes ${ }^{2}$.

La relativité du sens des mots compris dans le lexique socio-politique a aussi été signalée, d'une façon plus scientifique, par des linguistes appartenant tant au monde capitaliste qu'au monde socialiste. Par exemple :

The two great linguistic associations in East and West run foul of each other owing to mutual incomprehension. They accuse each other of having no democracy and no freedom; and democracy and freedom are among the signs that when analysed within the given sign system can be shown to have utterly different semantic contents in the two associations ${ }^{2}$.

The connotation of meaning in a number of units of the sociopolitical vocabulary is revealed in the specific conditions of the development of certain divided nations [il fait allusion au cas des deux Allemagne] or of class inequality in society. The absence of an explicit expression of the Weltanschauung (in the morphological structure of the word) is by no means equivalent to the absence of connotation. Connotation is formed as the word is used by different social groups under different historical and social conditions. Thus the word is accompanied or may be accompanied not only by the thought of the directly denoted object but also by indirectly connoted phenomena : ideas, feelings, cultural and situational circumstances, and others. Sometimes this connotation is minimal, but in certain cases it is so hypertrophied that it gives some investigators occasion to even speak of the absence of meaning as such in quite a number of words in certain texts ${ }^{8}$.

Une étude sémantique exemplaire sur les changements historiques dans le sens du mot italien democrazia se trouvera dans Tullio de Mauro ${ }^{4}$.

1. Il y a plus d'un quart de siècle, George Orwell stigmatisait dans Nineteen Eighty-four la distorsion du langage comme outil de propagande dans un Etat totalitaire. Il analyse lucidement dans un appendice à son roman les procédés que la propagande emploie pour vider de son sens le langage ordinaire, et moyennant ces procédés, pour manier les consciences :

The purpose of Newspeak was not only to provide a medium of expression for the world-view and mental habits proper to the devotees of Ingsoc [c'-à-d., le système au pouvoir], but to make all other modes of thought impossible... Its vocabulary was so constructed as to give exact and often very subtle expression to every meaning that a Party member could properly wish to express, while excluding all other meanings and also the possibility of arriving at them by indirect methods. This was done partly by the invention of new words, but chiefly by eliminating undesirable words and by stripping such words as remained of unorthodox meanings, and so far as possible of all secondary meanings whatever. To give a single example. The word free still existed in Newspeak, but it could only be used in such statements as \&This $\operatorname{dog}$ is free from lice» or "This field is free from weeds 》. It could not be used in its old sense of \& politically free » or \& intellectually free », since political and intellectual freedom no longer existed even as concepts, and were therefore of necessity nameless (G. Orwell, Nineteen Eighty-four, Toronto, Sanders \& Co., 1949, p. 303).

2. Louis Hjelmslev, The content form of language as a social factor $\$$, dans Essais linguistiques, Copenhague, Nordisk Sprog-og Kulturferlag, $1959, \mathrm{p}: 94$ :

3. N.G. Konlev, Components of the Content Stracture of the Word, The Hague-Paris, Mouton, 1976, p. 147-148.

4. Tullio de Mauro, Senso e significato, Bari, Adriatica Editrice, 1971. 


\section{Les dilemmes du traducteur}

Le problème que nous voulons poser dans cet article est celui du dilemme déontologique et idéologique du traducteur. D'abord, c'est le problème de nature technique qui se pose : quel procédé faudra-t-il employer pour transmettre fidèlement et exhaustivement le contenu d'un texte et des présupposés idéologiques qui constituent son contexte, à des lecteurs peu familiarisés avec la réalité sociale et historique concrète là où ce texte a été produit ?

Mais après, c'est une question de responsabilité du traducteur : que doit-il faire quand sa version, même si elle est heureuse, lui révèle pourtant une distorsion dans la terminologie ou dans les présuppositions et le place devant la situation d'avaliser le contenu de vérité du texte alors qu'il a pleine conscience que les mots altèrent ou faussent la réalité des faits?

Le traducteur, a-t-il le droit de mettre en jeu ses convictions personnelles pour avertir le lecteur que ce texte lui paraît faux ou piégé, et cela sans compromettre l'objectivité qui doit être à la base de son travail ?

\subsection{Le probleme technique}

\subsubsection{Le sens du texte source}

Le premier pas consiste à préciser le sens du texte source, c'est-à-dire non seulement la somme des signifiés des mots qui le constituent, mais aussi l'ensemble des circonstances qui, dans l'acte concret de communication, établissent un rapport entre le texte et son emploi. Cet ensemble de circonstances qu'on peut appeler contexte (où interviennent tous les éléments de la communication : émetteur, référent, code, canal du message) donne un sens particulier au discours. Non seulement les textes - écrits ou oraux - constituent le discours, mais l'ensemble d'actes et de matières signifiantes productrices de sens (comportements, rites, accumulation et échange d'objets, etc.), de façon que dans les conditions sociales de production, circulation et consommation des discours, dans ce sens large, on puisse fonder une sémiotique de l'idéologie et du pouvoir ${ }^{5}$.

Si l'idéologie est définie comme «système de rapports entre un ensemble signifiant donné et ses conditions sociales de production» (Veron, p. 14), les effets d'un discours donné à l'intérieur d'un tissu déterminé de rapports sociaux posent la question du pouvoir de la parole. Cette affirmation est encore plus évidente dans un État totalitaire, mais sa valeur reste identique dans n'importe quelle société : les maîtres du pouvoir ne sont pas seulement maîtres de la force et/ou de l'argent, mais aussi maîtres de la parole. Et le discours politicojuridique sacralise la notion de pouvoir, justifie l'idéologie dominante, impose un sens canonique au code linguistique et, le cas échéant, détruit les significations originaires si cela devient nécessaire pour la consécution de ses objectifs. Il utilise ce pouvoir pour vider de contenu les discours antagoniques et pour les transformer ainsi en nouveaux messages, parfois brutalement contradictoires.

5. Voir Eliseo Veron, « Sémiosis de l'idéologie et du pouvoir » dans Communications, 28 (1978), Paris, Seuil. 
C'est le cas des devises forgées par le Minitrue (Ministère de lá Vérité) de Nineteen Eighty-four : «La guerre est la paix », «La liberté est esclavage ». Ces paradoxes grotesques deviennent malheureusement de plus en plus une vérité amère dans nombre de sociétés contemporaines.

\subsubsection{Le poids du contexte historique}

Le discours juridico-politique reflète, comme toute manifestation de la super-structure sociale, non seulement les rapports de production au niveau de la structure socio-économique dominante à un moment donné, mais aussi un lest de structures caduques ou décadentes. Dans les processus de changement institutionnel, à l'intérieur de l'organisation d'un État, le législateur s'efforce de se dépouiller des sens caducs des discours antérieurs et il trouve des difficultés à forger de nouveaux sens dans le cadre du discours traditionnel. Les innovations et même les violations du code établi sont précisément des clés pour mesurer l'ampleur des changements que l'on essaie d'imposer.

Dans les processus révolutionnaires, tout comme dans les régimes restaurateurs d'ordres périmés, le souci de mettre en relief les antagonismes et les contradictions avec les systèmes que l'on veut remplacer ou modifier est réel. Cependant, un nouveau problème se pose dans le cas des régimes militaires qui, dans les dernières décennies, ont fait leur apparition sur la scène politique de l'Amérique latine : le discours officiel ne souligne pas toujours un changement, bien au contraire il s'approprie le lexique des discours politiques antérieurs pour les vider de contenu et infiltrer à la place des connotations idéologiques différentes.

Depuis la période des guerres de l'Indépendance dans les anciennes colonies espagnoles et portugaises (1810-1824), les régimes militaires ont pris la relève de façon continue des gouvernements civils constitutionnellement élus. Les coups d'État sont devenus si fréquents qu'ils ont fini par être acceptés naturellement, comme des faits inévitables du devenir politique, à tel point que le droit constitutionnel latino-américain les entérine avec la doctrine des « gouvernements de fait ». Ce qui veut dire qu'en l'absence des organes réguliers de l'État, les « gouvernements de fait » cumulent les pouvoirs législatif et exécutif et gouvernent à coups de décrets-lois qui peuvent - théoriquement - être annulés par un gouvernement constitutionnel postérieur. La constitutionnalité de ces décrets-lois peut être encore contestée par le pouvoir judiciaire, que les * gouvernements de fait »'osent généralement pas supprimer. Dans la pratique, les «gouvernements de fait » latino-américains ont agi d'habitude impunément, comme des dictatures sans freins, et leurs actions ont été légalisées par des tribunaux complaisants.

Mais cette faiblesse de la structure juridique du \& gouvernement de fait \$ - l'éventuelle annulation de son corps législatif par un pouvoir légitime ou par une justice inébranlable - ne convenait pas aux régimes militaires surgis des coups d'Etat des dernières décennies (à partir du coup d'Etat brésilien de 1964). Dans leurs actions s'inscrit la volonté de concentrer la totalité du pouvoir politique et encore de s'octroyer le pouvoir constitutionnel. C'est pour cela 
qu'ils justifient le coup d'État (pronunciamiento) en élaborant des actes, des statuts et des proclamations institutionnelles, qu'ils placent au premier plan de l'organisation juridique de l'État en même temps qu'ils déclarent vouloir respecter les constitutions nationales en ce qu'elles ne s'opposent pas à ces * principes révolutionnaires». Principes qui justifient l'usurpation du pouvoir. Il est très important d'analyser le langage de ces pronunciamientos pour comprendre l'ampleur de la déviation qui peut atteindre l'usage du vocabulaire juridico-politique au service d'une idéologie qui se propose d'annuler l'ordre juridique.

\section{Une étude de cas : les documents du coup d'État argentin de 1976}

$\mathrm{Au}$ cours des cinquante dernières années, l'armée argentine a interrompu l'ordre constitutionnel en six occasions : 1930, 1943, 1955, 1960, 1966 et 1976, sans compter les soulèvements étouffés ni les remplacements d'un président militaire par un autre. Dans les quatre premiers cas, les protagonistes du coup d'Etat annonçaient leur intention de corriger des erreurs ou des vices du gouvernement constitutionnel renversé et se présentaient comme un gouvernement transitoire en même temps qu'ils promettaient des élections. Dans les deux derniers cas, les trois armées (de terre, de mer et de l'air) ont assumé la responsabilité du coup d'État et ont installé à la place du gouvernement des juntes militaires où l'équilibre des trois forces pesait plus que la personnalité de ses membres. Elles ont aussi désigné un chef de gouvernement militaire, sans fixer de terme à son mandat. La «Révolution argentine» qui a mené au pouvoir entre 1966 et 1973 les généraux Onganía, Levingston et Lanusse, fut obligée par la pression sociale à convoquer des élections et à rendre le pouvoir au parti triomphant. Le dernier coup d'Etat s'interdit de se nommer « révolution 》 et essaie de diluer au maximum la responsabilité de l'interruption de l'ordre constitutionnel. Il se donne le nom de "Processus pour la Réorganisation nationale», son chef formel depuis deux ans étant le général Videla, qui vient de prendre sa retraite. Il reste à la tête de l'exécutif comme président civil, à côté de la Junte militaire. On parle d'un nouveau «modèle de pouvoir», dont Videla est le "quatrième homme ", mais ce modèle s'est déjà montré apte à produire des conflits de protocole, lorsqu'il s'agit de préciser s'il faut placer la Junte en avant, en arrière ou sur le même rang que le président de l'exécutif.

Nous avons jugé utile de présenter quelques passages, les plus représentatifs, des documents juridiques du «Processus» dans une version la plus littérale possible, afin de montrer les difficultés que leur interprétation pose à un lecteur qui ignorerait les coulisses de l'histoire politique argentine.

\subsection{Acte pour le processus de Réorganisation nationale}

Vu l'état actuel du pays, les commandants militaires décident de se constituer en Junte militaire, laquelle :

1) Assume le pouvoir politique de la République.

2) Déclare la caducité des mandats du président de la Nation, des gouverneurs, et des vice-gouverneurs des provinces. 
3) Ordonne la cessation dans leurs fonctions des commissaires fédéraux des provinces sous tutelle, du gouverneur de la Terre de Feu, de l'Antarctique et des îles de l'Atlantique méridional et du maire de la ville de Buenos Aires.

4) Dissout le Congrès national, les Législatures provinciales, la Salle des Représentants de Buenos Aires et les Conseils municipaux des provinces ou d'autres organismes similaires.

5) Déplace les membres de la Haute Cour de Justice de la Nation, le procureur général de la Nation et

6) les membres des Hautes Cours des provinces, ainsi que le procureur da Trésor.

7) Suspend l'activité des partis politiques, ainsi que

8) l'activité syndicale des travailleurs, entrepreneurs et professionnels.

9) Notifie ces actes aux représentations diplomatiques aux fins d'assurer la continuité des rapports internationaux.

10) Une fois ces mesures accomplies, elle désigne le citoyen qui exercera la présidence de la Nation.

11) Dispose que les commissaires militaires agiront dans leurs juridictions respectives à l'instar de ce qui a été établi dans la juridiction nationale et d'après les instructions données par la Junte militaire.

3.2 Acte de propos et d'objectifs fondamentaux pour le Processus de Réorganisation nationale

1) Propos : Restituer les valeurs essentielles qui sont à la base de la condition intégrale de l'État, en mettant l'accent sur la moralité, la capacité et l'efficience, indispensables à la reconstruction du contenu et de l'image de la Nation; déraciner la subversion et promouvoir le développement économique de la vie nationale, fondé sur l'équilibre et la participation responsable des différents secteurs afin d'assurer l'instauration postérieure d'une démocratie républicaine, représentative et fédérale appropriée à la réalité et aux exigences de solution et de progrès du peuple argentin.

2) Objectifs fondamentaux :

2.1) Réalisation d'une souveraineté politique assise sur des institutions constitutionnelles revivifiées, capables d'élever l'intérêt national au-dessus des sectarismes, des tendances ou des personnalismes.

2.2) Mise en vigueur des valeurs de la morale chrétienne, de la tradition nationale et de la dignité de l'être argentin.

2.3) Mise en vigueur de la sécurité nationale, en déracinant la subversion et les causes qui favorisent son existence, 
2.4) Mise en pleine vigueur de l'ordre juridique et social.

2.5) Réalisation d'une situation socio-économique, capable d'assurer la capacité de décision nationale et la pleine réalisation de l'homme argentin, où (sic) l'État maintiendra le contrôle sur les aires vitales qui commandent la sécurité et le développement et offrira à l'initiative et aux capitaux privés et étrangers les conditions nécessaires à une participation fluide dans le processus d'exploitation rationnelle des ressources en neutralisant toute possibilité d'interférence de ces capitaux dans l'exercice des pouvoirs publics.

2.6) Obtention du bien-être général au moyen du travail fécond en égalité d'opportunités et en appliquant des critères adéquats de justice sociale.

2.7) Rapports harmonieux entre l'Etat, le capital et le travail, avec un développement vigoureux des structures patronales et syndicales restreintes à leurs fins spécifiques.

2.8) Formation d'un système éducatif adapté aux besoins du pays, utile aux objectifs de la Nation et capable de consolider les valeurs et les aspirations culturelles de l'être argentin.

2.9) Position internationale dans le monde occidental et chrétien, tout en maintenant la capacité d'auto-détermination et en assurant une présence argentine dans le concert des nations.

\subsection{Commentaire}

Le premier document est de nature exécutive : les mots clés sont les verbes performatifs, qui portent le poids sémantique de l'acte illocutoire d'affirmer l'autorité de la Junte militaire. Celle-ci «assume le pouvoir», et en conséquence «déclare», «ordonne », « dissout », " déplace», « suspend», « notifie », « désigne », « dispose ». Pour juger de la légitimité de ces actes il ne faut pas oublier qu'un acte illocutoire est sujet à la condition que son énonciation ait pour fonction spécifique d'accomplir cette action par le fait même de l'énoncer et que l'émetteur possède l'autorité reconnue pour l'accomplir ${ }^{6}$.

Malgré son souci de maintenir ses actions dans un cadre juridique, malgré la légitimation accordée par un pouvoir judiciaire ad hoc, la Junte militaire ne peut pas cacher l'illégitimité de ses origines : les commandants militaires se sont érigés en autorités et personne ne leur a donné mandat pour ce faire, même s'ils déclarent avoir reçu le mandat de "leurs forces» (leurs subordonnées!). Ceci constitue une violation flagrante de l'article 21 de la Constitution : \& Le peuple ne délibère et ne gouverne que par l'intermédiaire de ses représentants. Toute armée ou réunion de personnes qui assume les droits du peuple et pétitionne en son nom, commet le délit de sédition. »

Les autres dispositions de l'Acte pour le Processus sont également frappées de nullité à l'intérieur du système constitutionnel. Les autorités - exécutives

6. La théorie des actes illocutoires a été surtout développée par les «philosophes du langage ordinaire » de l'école d'Oxford, à partir des réflexions de J.-L. Austin. Pour un exposé d'ensemble de la théorie, voir J.R. Searle, les Actes de langage. Essai de philosophie du langage, Paris, Hermann, 1972. 
ou judiciaires - peuvent être jugées en « jugement politique » seulement par le Congrès de la Nation. Ni celui-ci ni les autres organes délibératifs provinciaux ou municipaux ne peuvent être dissous par une autorité centrale. Les membres $\mathrm{du}$ pouvoir judiciaire sont inamovibles tant que leur conduite est irréprochable. Le président de la Nation doit être élu au suffrage universel. Et ainsi de suite. C'est-à-dire que l'Acte pour le Processus est inconstitutionnel du début à la fin. En outre, «l'état actuel du pays » auquel font appel les commandants des forces armées pour justifier le coup d'État ne constituait pas la figure constitutionnellement prévue d'une situation d'urgence ni d'extrême détresse, puisque dans une année il y aurait eu des élections générales.

On peut considérer maintenant le degré de véracité du deuxième document à la lumière de ces circonstances. Signalons que son contenu vise à exposer les raisons du coup d'État militaire. L'analyse des propos et des objectifs nécessite une méthodologie capable de montrer le sens du texte dans les circonstances où il a été publié : immédiatement après le coup d'État. L'intention était d'obtenir le consensus de la population pour y fonder une légalité appuyée sur des principes d'éthique et des vertus politiques. La véritable portée de ce texte et la façon dont la Junte l'a utilisé pour affirmer son autorité peuvent être mises en lumière si on le soumet à une analyse des présuppositions et de l'intentionalité.

Le «propos» vise à louer les vertus d'un futur gouvernement conduit par des militaires. Il dénigre par contraste implicite, les vices du gouvernement civil renversé. «Restituer » implique que les valeurs essentielles avaient été oubliées. «En mettant l'accent \accuse d'une façon voilée le gouvernement destitué d'immoralité, de manque d'aptitude et d'inefficacité. « Reconstruction » présuppose qu'on a détruit « le contenu et l'image de la Nation» (?). "Déraciner la subversion » affirme qu'il y a des germes de dissolution enracinés dans la société et soupçonne par la suite tous les adversaires potentiels de la Junte d'être factieux ou délinquants. "L'équilibre et la participation responsable » dénonce vaguement tous les secteurs ( $62 \%$ de la population) qui avaient voté pour le gouvernement précédent, et même l'opposition politique qui, jusqu'à la dernière minute, avait défendu l'ordre constitutionnel. Tous ces partis populaires sont qualifiés d'un seul coup de déséquilibrés, d'apathiques ou d'irresponsables. Enfin, l'expression sert à déclarer que le but de « Processus » est d'instaurer une démocratie républicaine, représentative et fédérale, en même temps qu'elle met en doute l'histoire politique du pays, en exigeant que cette démocratie soit * adéquate à la réalité et aux exigences... du peuple argentin ». Qui pourrait être contre cette dernière condition? Le problème réside dans ce fait que seule la Junte militaire a le droit de vote pour décider quelle est la forme républicaine, représentative et fédérale qui convient au peuple argentin, et du moment où l'instaurer.

Avec les « objectifs fondamentaux » une technique de la manipulation des présuppositions est évidente : l'en-tête de chaque alinéa est constitué par un nom destiné à "confirmer», disons, les principes et les valeurs qui ont toujours été inscrits comme des aspirations dans la vie politique argentine. Le document pré- 
suppose que ces principes et valeurs n'ont jamais été pratiqués, mais seulement déclamés, et, en passant, il implique que le gouvernement qui a produit ce document changera enfin en réalité ces aspirations longuement caressées.

Si ces principes ont été énoncés il y a plus d'un siècle dans la Constitution nationale (1853), et s'ils ont été répétés avec plus ou moins de vigueur dans les programmes et les manifestes de la plupart des partis, mouvements et tendances politiques démocratiques de l'Argentine pendant tout ce siècle, où réside alors la nouveauté des « objectifs fondamentaux» et pourquoi les nouvelles autorités croient-elles nécessaire de les expliciter?

C'est que, subtilement, la Junte militaire s'est approprié ces principes en exclusivité, et par son refus de les partager avec les forces politiques déjà existantes, celles-ci se trouvent automatiquement disqualifiées et placées en opposition, voire accusées de délinquance subversive.

Analysons les présuppositions :

2.1) Les gouvernements précédents n'ont pas su exercer la souveraineté politique, ou bien ils l'ont assise sur des institutions périmées, ou bien ils ont préféré des intérêts sectaires, tendancieux ou personnalistes à l'intérêt national. Tous ont donc été antinationaux.

2.2) Les gouvernements précédents n'ont respecté ni la morale chrétienne, ni la tradition nationale, ni la dignité de l'être argentin : ils ont tous été immoraux, dégradés et corrompus.

2.3) Les gouvernements précédents ont mis en danger la sécurité nationale, en tolérant la subversion (concept très large, qui comprend tant la résistance armée aux autorités établies que l'infiltration $d^{\text {' } ~ \& i d e ́ e s ~ d a n g e r e u s e s ~ 》) ~ o u ~ b i e n ~}$ parce qu'ils n'ont pas su résoudre les problèmes de la misère, du chômage, etc. Ils ont ainsi donné lieu à la naissance du mécontentement et du désaccord.

2.4) Les gouvernements précédents ont violenté ou laissé violenter l'ordre juridique et altéré ou laissé altérer l'ordre social : ils ont donc été ou bien arbitraires et factieux ou bien faibles et incompétents.

2.5) Les gouvernements précédents n'ont pas résolu les problèmes socio-écomiques du pays d'une façon satisfaisante : ils l'ont livré à la dépendance des intérêts extra-nationaux ou bien ils ont permis l'exploitation des citoyens. Quant au problème des capitaux étrangers - problème politique extrêmement épineux dans le Tiers Monde - les implications sont ambiguës : les gouvernements précédents ont tour à tour fait obstacle aux investissements bienveillants ou accepté de bon gré qu'ils agissent malhonnêtement sur les pouvoirs publics.

2.6) Les gouvernements précédents n'ont pas promu le bien-être général, ou bien ils l'ont promis par démagogie, ils en ont fait cadeau, en provoquant ainsi des déséquilibres et des inégalités dans la population. La justice sociale, un des étendards du gouvernement péroniste renversé, n'est pas condamnée comme principe, mais on considère que sa mise en pratique était viciée de démagogie.

2.7) Les gouvernements précédents n'ont pas réussi à équilibrer harmonieusement le capital et le travail, et pire encore, ils ont accepté et même promu l'ingé- 
rence des associations patronales et des syndicats dans la vie politique. C'est l'État, donc, qui doit s'occuper de régler leurs activités.

2.8) Le système éducatif ne répond pas aux besoins du pays, parce que inefficace et caduc, mais surtout parce que l'école a contribué à la diffusion de doctrines exotiques et anti-nationales.

2.9) La diplomatie argentine a été autonome, mais elle doit continuer, comme à l'époque de la guerre froide, de s'aligner sur le bloc « occidental et chrétien ». Les gouvernements précédents ont flirté parfois avec des pays socialistes ou du Tiers Monde, et cela risque d'affaiblir le prestige international de l'Argentine.

\section{Le problème déontologique}

Bref, les responsables du \& Processus» se permettent de juger le passé, le présent et le futur de la vie politique, constitutionnelle et juridique du pays dans des documents législatifs, dans des textes normatifs. Leur responsabilité personnelle diluée dans l'engagement patriotique des forces armées, les trois commandants (qui, d'autre part, sont relevés périodiquement) ont acquis ainsi le privilège de faire passer leurs opinions et celles de leurs camarades d'armes par des jugements historiques. S'ils s'agissait de citoyens ordinaires, leurs pensées n'auraient pas eu plus de poids que celles des autres citoyens. Ce sont souvent des parti pris, des engagements avec une idéologie de classe. En effet, on peut y reconnaître des traits d'une pensée bourgeoise, moyennement conservatrice, avec quelques indices de libéralisme progressiste d'une part, et certains principes du nationalisme catholique d'autre part: il s'agit d'un compromis entre différentes tendances idéologiques très répandues dans la haute société argentine et aussi dans les forces armées. Simples opinions, mais revêtues de la force illocutoire que leur confère le fait d'avoir été proclamées du sommet du pouvoir, elles sont devenues des lois, plus encore, la loi suprême de la Nation.

Le traducteur, peut-il les traduire comme s'il s'agissait d'un texte juridique quelconque provenant d'une autorité compétente ? Ou bien est-il tenu d'avertir les lecteurs des circonstances qui ont fait naître ce texte, de signaler ou de souligner les présuppositions et les implications que le texte contient?

Nous avons choisi de poser le problème au niveau des présuppositions linguistiques déductibles du texte, sans toucher aux contradictions entre ces énoncés et les actions d'un régime dictatorial. En effet, on ne pose pas ici la question de savoir si le traducteur est autorisé ou non à évaluer le texte à partir de ses informations sur la réalité historico-sociale, celle d'où ce texte provient. Il ne s'agit pas de suggérer, par exemple, que le traducteur rejette l'expression "développement vigoureux des structures syndicales » parce qu'il sait que la plupart des activités syndicales sont interdites en Argentine depuis le coup d'État et que bon nombre de syndicalistes sont en prison ou sont portés disparus ou assassinés. Ce problème touche directement la question de la vérité matérielle de l'information, des rapports entre le message et son référent, mais nous croyons que le traducteur ne doit pas, à ce niveau, s'attribuer le rôle de médiateur entre le texte et le lecteur, à moins qu'il ne soit en même temps un commentateur de 
l'information contenue. Ce que nous voulons soulever ici c'est le problème du traitement des présuppositions comprises dans le texte même, parce que nous croyons que le traducteur en est aussi responsable, et qu'il ne peut pas se prévaloir des principes d'objectivité et de neutralité professionnelle pour ignorer ce problème.

Ainsi, si le traducteur avertit, par exemple, que l'expression « la pleine vigueur de l'ordre juridique » est un paradoxe dans un document émis par une autorité inconstitutionnelle, doit-il ou non prévenir le lecteur? S'il comprend que «subversif » est dans le code employé comme une notion qui comprend les hauts fonctionnaires des gouvernements précédents (même un président de la République et les gouverneurs des provinces légitimement êlus par une large majorité des suffrages), peut-il continuer de traduire ce mot implicitement ou explicitement associé au mot «délinquants», quand ces gens-là n'ont jamais été soumis à un procès par des tribunaux compétents?

Admettre cet élargissement de la responsabilité du traducteur par rapport aux contenus de sa version signifie que l'on pose une fois encore la question du principe d'objectivité, ou plus précisément la question des limites de la participation du traducteur dans la composition du texte. Nous sommes conscients que pour ce type de discours il est bien difficile de tracer les limites, mais nous croyons aussi que si le traducteur, en appliquant ses connaissances linguistiques et son expérience, peut démontrer les paradoxes, les ambiguïtés, les contradictions d'un texte, il doit le signaler par le moyen le plus adéquat. Le mieux serait, probablement, de ne pas toucher mais d'y ajouter des notes. Ces notes rempliraient la fonction d'expliciter les présuppositions qui, naturellement, ne peuvent pas être pleinement maintenues quand on déplace un discours politique de la société où il a été produit, parce que, reprenant les mots de Oswald Ducrot, «quant au discours politique, il en resterait fort peu de chose si l'on en retirait les présupposés ? $\gg$.

ENRIQUETA RIBÉ Miguel Olivera

7. O. Ducrot, Dire et ne pas dire. Principes de sémantique linguistique, Paris, Hermann, 1972. C'est une étude en profondeur des rapports implicites dans l'acte d'énonciation. 